Original Paper http://ajol.info/index.php/ijbcs http://indexmedicus.afro.who.int

\title{
Le profenofos, un alternatif à l'endosulfan en culture cotonnière au Bénin
}

\author{
Cocou A. DJIHINTO ${ }^{1 *}$, Antoine AFFOKPON ${ }^{1}$, Elie DANNON ${ }^{2}$ et Gustave BONNI ${ }^{1}$ \\ ${ }^{1}$ Institut National des Recherches Agricoles du Bénin, 01BP 884 Cotonou, République du Bénin. \\ ${ }^{2}$ International Institute of Tropical Agriculture (IITA), 08-B.P. 0932, Tri Postal Cotonou, Bénin. \\ *Auteur correspondant, E-mail : djihinto@yahoo.com; Tel : 0022997983485
}

\section{RESUME}

Dans l'objectif de trouver un alternatif à l'endosulfan, l'efficacité du profenofos et de l'endosulfan a été étudié dans un dispositif de bloc aléatoire complet en station et en milieu réel. Sur station, le profenofos assure un contrôle, équivalent et parfois significativement supérieur $(\mathrm{p}<0,001)$ à l'endosulfan, des populations des ravageurs comme Helicoverpa armigera, Earias spp, Pectinophora gossypiella, Cryptophlebia leucotreta. Le Fanga $500 \mathrm{EC}$ (profenofos $750 \mathrm{~g} / \mathrm{ha}$ ) a été significativement supérieur $(\mathrm{p}<0,05)$ à Callisulfan $350 \mathrm{EC}$ (endosulfan $700 \mathrm{~g} / \mathrm{ha}$ ) pour le contrôle des populations de Aphis gossypii, Pectinophora gossypiella et Earias spp. Il en résulte une production de capsules vertes saines qui montre que le Calfos 500 EC (profenofos 750 $\mathrm{g} / \mathrm{ha}$ ) est équivalent aux différentes formulations d'endosulfan testées et parfois significativement meilleur ( $\mathrm{p}<$ 0,01) au Thiofanex 350 EC (endosulfan $700 \mathrm{~g} / \mathrm{ha}$ ). A la récolte du coton graine, le profenofos procure un rendement significativement supérieur $(\mathrm{p}<0,05)$ à l'endosulfan. En milieu paysan, aucune différence significative $(\mathrm{p}>0,05)$ n'a été observée entre le Calfos $500 \mathrm{EC}$ (profenofos $750 \mathrm{~g} / \mathrm{ha}$ ) et le Callisulfan $350 \mathrm{EC}$ (endosulfan $700 \mathrm{~g} / \mathrm{ha}$ ) à la récolte du coton graine. Le profenofos peut être appliqué comme un alternatif à l'endosulfan en culture cotonnière au Bénin.

() 2016 International Formulae Group. All rights reserved.

Mots clés : Profenofos, alternatif à l'endosulfan, culture cotonnière, Bénin.

\section{The profenofos, an alternative to endosulfan in cotton crop in Benin}

\begin{abstract}
In the objective to find an alternative to endosulfan, the efficacy of profenofos and endosulfan has been studied in a complete randomized block design in experimentation station and in real environment. In station, the profenofos provides, in comparison with endosulfan, an equivalent control, and sometimes, a significantly higher $(\mathrm{p}<0.001)$ control, of cotton pest populations including Helicoverpa armigera, Earias spp, Pectinophora gossypiella, Cryptophlebia leucotreta. The effect of Fanga 500 EC (profenofos $750 \mathrm{~g} / \mathrm{ha}$ ) has been significantly higher ( $\mathrm{p}<0.05$ ) than the effect of Callisulfan $350 \mathrm{EC}$ (endosulfan $700 \mathrm{~g} / \mathrm{ha}$ ) for the control of the populations of Aphis gossypii, Pectinophora gossypiella and Earias spp. It results, of this fact, a production of the best quality of cotton which shows that the Calfos $500 \mathrm{EC}$ (profenofos $750 \mathrm{~g} / \mathrm{ha}$ ) is equivalent to various formulations of endosulfan tested and sometimes significantly better $(p<0.01)$ than Thiofanex 350 EC (endosulfan $700 \mathrm{~g} / \mathrm{ha}$ ). At harvest time, the profenofos provides a yield of seed cotton significantly higher $(p<0.05)$ than those obtained with endosulfan. In real environment, at harvest time of seed cotton, no
\end{abstract}


significant difference ( $p>0.05$ ) has been observed between the Calfos $500 \mathrm{EC}$ (profenofos $750 \mathrm{~g} / \mathrm{ha}$ ) and the Callisulfan $350 \mathrm{EC}$ (endosulfan $700 \mathrm{~g} / \mathrm{ha}$ ). The profenofos can be used as an alternative to endosulfan in cotton crop in Benin.

(c) 2016 International Formulae Group. All rights reserved.

Keywords: Profenofos, alternative to endosulfan, cotton crop, Benin.

\section{INTRODUCTION}

Le cotonnier est une plante hôte qui héberge plusieurs ravageurs de la graine jusqu'à l'ouverture des capsules en passant par la levée, la floraison et la fructification. Une dizaine d'espèces parmi ces ravageurs présentent une réelle importance économique au Bénin (Katary, 2003; Djihinto, 2004). Durant la phase reproductive, les boutons floraux, les fleurs et les capsules sont attaqués par un groupe de chenilles appartenant aux genres Helicoverpa, Earias, Diparopsis, Pectinophora et Cryptophlebia (Katary, 2003 ; Djihinto, 2004).

Plusieurs méthodes de luttes sont utilisées pour la protection phytosanitaire de cette culture. Sur le plan variétal, la variété pileuse recommandée par la recherche et adoptée par les paysans au Bénin protège la culture contre les Jacides (Katary, 2003; Djihinto, 2004 ; Prudent et al., 2007). La lutte agronomique repose sur la fertilisation équilibrée, la date et la densité des semis. La lutte biologique utilise naturellement les auxiliaires comme les coccinelles prédatrices qui réduisent les populations des pucerons (Katary, 2003 ; Djihinto, 2004 ; Prudent et al., 2007). La protection chimique est au centre de toutes ces techniques phytosanitaires au Bénin.

Compte tenu du complexe de ravageur inféodé à cette culture, la stratégie classique de protection du cotonnier au Bénin est basée sur six applications insecticides à partir de 50 jours après la levée de la culture. Deux applications insecticides successives sont espacées de 14 jours (Katary, 2003 ; Djihinto, 2004 ; Katary et Djihinto, 2007). Plusieurs matières actives sont utilisées en mélange (Katary, 2003 ; Djihinto, 2004 ; Katary et
Djihinto, 2007). Les pyréthrinoïdes, les organophosphorés potentialisants aphicides et acaricides sont utilisés respectivement contre les carpophages, les piqueurs suceurs et les acariens ou phyllophages (Djihinto, 2004 ; Silvie et al., 2013).

L'avènement de la résistance de Helicoverpa armigera aux pyréthrinoïdes vers la fin des années 1990 (Djihinto et al., 2009, $2012,2013)$ a marqué un tournant décisif dans la lutte chimique en culture cotonnière, en Afrique de l'Ouest en général et au Bénin en particulier, avec l'utilisation massive de l'endosulfan à forte dose (660 à 750 gramme de matière active à l'hectare) aux deux premiers traitements du cotonnier pour maitriser les infestations de ce ravageur. Les deux traitements suivants utilisent de mélange pyréthrinoïde et organophosphoré (OP) acaricide et les deux dernières applications utilisent de mélange pyréthrinoïde et OP aphicide (Katary, 2003 ; Djihinto, 2004 ; Katary et Djihinto, 2007; Silvie et al., 2013).

Compte tenu de la toxicité relative de l'endosulfan et du phénomène de résistance de certains insectes à cet insecticide, cette molécule a été retirée du programme de protection des cultures en général et de la protection phytosanitaire du cotonnier en particulier vers la fin des années 2000 (Karim, 2000 ; Kathuria et al., 2005 ; Yaqoob et Arora, 2005; Nimbalkar et al., 2009; Agbohessi et al., 2012 ; Djihinto et al., 2013, 2012, 2009). Face à cette situation, la recherche d'alternative à l'endosulfan s'avère nécessaire. Des études antérieures ont testé au Bénin et dans d'autres pays, plusieurs molécules alternatives à l'endosulfan comme le profenofos, l'indoxacarb, le spirotétramate- 
flubendiamide, le spinosad, l'emamectine, le lufenuron qui sont appliqués sur de nombreuses cultures dont le coton (Ochou et Martin, 2002 ; Martin et al., 2002 ; Djihinto, 2004 ; Cheema et al., 2004 ; Kathuria et al., 2005 ; Razaq et al., 2005 ; Katary et Djihinto, 2007 ; Dastjerdi et al., 2008 ; Agbohessi et al., 2012). Dans l'objectif d'élargir la gamme des produits alternatifs à l'endosulfan, la présente étude a été consacrée à la comparaison de l'efficacité du profenofos et de l'endosulfan vis-à-vis du complexe des ravageurs du cotonnier au Bénin.

\section{MATERIEL ET METHODES \\ Matériel végétal}

La variété H279-1 du cotonnier Gossypium hirsutum a été utilisée dans le cadre de ce travail.

\section{Insecticides \\ Endosulfan}

Les formulations EC (Emulsifiable Concentrate) et CS (Capsule Suspension) de l'endosulfan ont été expérimentées. Les produits commerciaux à base d'endosulfan testés ont été : Phaser 350 EC (endosulfan 350 $\mathrm{g} / \mathrm{l}$ ), Phaser $330 \mathrm{CS}$ (endosulfan $330 \mathrm{~g} / \mathrm{l}$ ), Thiofanex 350 EC (endosulfan $350 \mathrm{~g} / \mathrm{l}$ ), Callisulfan 350 EC (endosulfan $350 \mathrm{~g} / \mathrm{l}$ ). Les produits Phaser 350 EC et Phaser 330 CS provenaient de la Société Bayer (Allemagne) tandis que le Callisulfan $350 \mathrm{EC}$ et le Thiofanex 350 EC ont été fournis respectivement par les Sociétés Arysta Life Science (France) et Senchim (Sénégal).

\section{Profenofos}

$\mathrm{La}$ formulation EC (Emulsifiable Concentrate) de profenofos a été expérimentée. Les produits commerciaux à base de profenofos testés ont été le Calfos 500 EC (profenofos $500 \mathrm{~g} / \mathrm{l}$ ) et le Fanga $500 \mathrm{EC}$ (profenofos $500 \mathrm{~g} / \mathrm{l}$ ). Le produit Calfos 500 EC appartenait à la société Arysta Life Science (France) et le Fanga 500 EC a été fourni par la société ALM (France).

\section{Méthodes}

Trois essais ont été installés dont deux essais sur station de recherche et un essai en milieu paysan.

\section{Essais sur station de recherche}

Pour les essais sur station de recherche, un dispositif de bloc aléatoire complet, comportant 6 répétitions et des parcelles élémentaires de 9 lignes de 20 mètres de longueur dont 8 lignes traitées, a été installé sur chacune des deux stations d'expérimentation du Sud et du Centre du Bénin, respectivement à Sékou et à Savalou.

Sur la station de Sékou, au Sud du Bénin, trois produits insecticides à base d'endosulfan ont été comparés à un insecticide à base de profenofos. Les doses des produits comparés ont été : Phaser 350 EC (endosulfan $700 \mathrm{~g} / \mathrm{ha}$ ), Phaser 330 CS (endosulfan $660 \mathrm{~g} / \mathrm{ha}$ ), Thiofanex $350 \mathrm{EC}$ (endosulfan $700 \mathrm{~g} / \mathrm{ha}$ ), Calfos 500 EC (profenofos $750 \mathrm{~g} / \mathrm{ha}$ ).

Au Centre du Bénin, sur la station de Savalou, deux produits insecticides à base de profenofos ont été comparés à un insecticide à base d'endosulfan. Les doses des produits testés ont été: Callisulfan 350 EC (endosulfan $700 \mathrm{~g} / \mathrm{ha}$ ), Calfos 500 EC (profenofos 750 g/ha), Fanga 500 EC (profenofos $750 \mathrm{~g} / \mathrm{ha}$ ).

Six applications des produits mis en comparaison ont eu lieu à partir de 50 jours après la levée du cotonnier. Deux applications insecticides successives ont été espacées de 14 jours. Sur chaque parcelle élémentaire, les observations suivantes ont été réalisées :

- Nombre de plants attaqués par Syllepte derogata, Aphis gossypii, Bemisia tabaci et Polyphagotarsonemus latus sur 20 plants observés une fois par semaine à partir de 49 jours après la levée du cotonnier,

- Nombre de feuilles attaquées par Aphis gossypii, Bemisia tabaci et Polyphagotarsonemus latus sur 100 feuilles observées une fois par semaine à partir de 49 jours après la levée du cotonnier,

- Nombre de chenilles de Helicoverpa armigera, Diparopsis watersi, Earias spp, Cryptophlebia leucotreta, Pectinophora 
gossypiella, Spodoptera littoralis sur 20 plants observés une fois par semaine à partir de 49 jours après la levée du cotonnier,

- Nombre de capsules vertes saines, trouées, piquées, pourries et nombre de chenilles rencontrées dans 100 capsules vertes observées une fois par semaine à partir de 84 jours après la levée du cotonnier,

- Nombre de capsules mûres saines, trouées, tachées, momifiées et proportion de coton jaune dans 100 capsules observées juste après chaque récolte de coton graine,

- Récolte du coton graine des deux lignes centrales.

\section{Essais en milieu paysan}

En milieu paysan, un essai a été installé dans le Centre du Bénin à Savalou. Un dispositif de bloc aléatoire complet, comportant 10 répétitions et des parcelles élémentaires de $1000 \mathrm{~m}^{2}$ entièrement traitées, a été installé.

Deux produits insecticides à base de profenofos ont été comparés à un insecticide à base d'endosulfan. Il s'agit de Callisulfan 350 EC (endosulfan $700 \mathrm{~g} / \mathrm{ha}$ ), Calfos $500 \mathrm{EC}$ (profenofos 750 g/ha), Fanga 500 EC (profenofos $750 \mathrm{~g} / \mathrm{ha}$ ).

En milieu paysan comme sur station, six applications insecticides ont été réalisées à partir de 50 jours après la levée du cotonnier. Deux applications insecticides successives ont été espacées de 14 jours. Les produits mis en comparaison ont été appliqués lors des deux premiers traitements du cotonnier. Dans chaque parcelle élémentaire, la récolte du coton graine a été effectuée sur 40 lignes dont chaque ligne mesure 20 mètres de longeur.

\section{Analyses statistiques}

Les données collectées ont été statistiquement analysées avec le logiciel STAT-ITCF (ITCF, Boigneville, France). Le test de Newman-Keuls a été utilisé pour comparer les moyennes des paramètres observés. Quand il y a une différence significative entre les moyennes des paramètres observés, les lettres $\mathrm{a}$, $\mathrm{b}$, et c sont utilisées pour faire le classement; ainsi les paramètres possédant la même lettre ne sont pas significativement différents.

\section{RESULTATS}

Essai sur station d'expérimentation dans le Sud Bénin à Sékou

Le Tableau 1 présente les résultats des comptages de chenilles carpophages observées sur les plants de cotonniers. Pour le contrôle de la chenille de $H$. armigera, le Calfos 500 EC (profenofos $750 \mathrm{~g} / \mathrm{ha}$ ), dans aucun cas, n'est significativement inférieur aux différentes formulations d'endosulfan testées. Au contraire le Calfos 500 EC est parfois significativement supérieur $(\mathrm{p}<0,001)$ au Thiofanex 350 EC (endosulfan $700 \mathrm{~g} / \mathrm{ha}$ ) et au Phaser 350 EC (endosulfan $700 \mathrm{~g} / \mathrm{ha}$ ). La même tendance a été observée pour le contrôle de Earias spp et c'est le Calfos 500 EC qui est parfois significativement supérieur ( $<<0,001)$ au Thiofanex 350 EC (endosulfan $700 \mathrm{~g} / \mathrm{ha}$ ) et au Phaser $350 \mathrm{EC}$ (endosulfan $700 \mathrm{~g} / \mathrm{ha}$ ). Dans le cas de la maîtrise des populations de Pectinophora gossypiella, le Calfos 500 EC et le Phaser 330 CS réduisent significativement mieux $(p<0,005)$ les populations de ce ravageur que ne le font le Phaser 350 EC et le Thiofanex 350 EC (Tableau 1).

Les Tableaux 2 et 3 présentent les pourcentages de capsules vertes saines, trouées et piquées déterminés dans cet essai. Les pourcentages de capsules saines indiquent que le Calfos 500 EC (profenofos $750 \mathrm{~g} / \mathrm{ha}$ ) est équivalent aux différentes formulations d'endosulfan testées et parfois significativement meilleur $(\mathrm{p}<0,01)$ au Thiofanex 350 EC (endosulfan $700 \mathrm{~g} / \mathrm{ha}$ ). On retrouve cette tendance lors du cinquième comptage des capsules vertes piquées et du septième comptage des capsules vertes trouées. L'équivalence du profenofos $750 \mathrm{~g} / \mathrm{ha}$ et parfois sa supériorité à l'endosulfan 700 g/ha (Thiofanex 350 EC) dans cet essai ont été aussi observées lors de l'examen des chenilles de Earias spp et C. leucotreta dans 100 capsules vertes (Tableau 3). Paradoxalement, la bonne efficacité du profenofos par rapport à 
l'endosulfan notée lors du dénombrement de P. gossypiella sur les cotonniers (Tableau 1) n'a pas été observée pendant le dénombrement du même ravageur dans les capsules vertes (Tableau 3); ceci peut s'expliquer par la très faible population de ce ravageur rencontré dans les capsules au niveau du Tableau 3 (en moyenne moins d'une chenille dans 100 capsules).

Enfin, à la récolte, seul le pourcentage de capsules mûres tachées est discriminant et le profenofos n'est inférieur à aucune formulation d'endosulfan testée (Tableau 3).

Essai sur station d'expérimentation et en milieu paysan dans le Centre Bénin à Savalou

Sur la station d'expérimentation et en milieu réel à Savalou, les paramètres observés et qui ont permis de discriminer l'endosulfan et le profenofos sont consignés dans les Tableaux 4 et 5 .

Le Calfos 500 EC et le Fanga 500 EC (profenofos $750 \mathrm{~g} / \mathrm{ha}$ ) ont été significativement meilleurs $(\mathrm{p}<0,005)$ à Callisulfan 350 EC (endosulfan $700 \mathrm{~g} / \mathrm{ha}$ ) durant le comptage de $P$. gossypiella sur les plants du cotonnier (Tableau 4).

L'efficacité des insecticides expérimentés vis-à-vis du contrôle des populations de A. gossypii montre un bon comportement du profenofos en comparaison avec l'endosulfan testé (Tableau 4). Le Fanga $500 \mathrm{EC}$ (profenofos $750 \mathrm{~g} / \mathrm{ha}$ ) a été toujours significativement supérieur $(\mathrm{p}<0,05)$ à Callisulfan 350 EC (endosulfan $700 \mathrm{~g} / \mathrm{ha}$ ) pour ce paramètre observé (Tableau 4).

Lorsqu'on examine les ravageurs dénombrés dans 100 capsules vertes (Tableau 5), malgré la faible population des chenilles rencontrées à l'intérieur des capsules, on s'aperçoit que le Fanga 500 EC (profenofos $750 \mathrm{~g} / \mathrm{ha}$ ) contrôle mieux les infestations de $P$. gossypiella et Earias spp par rapport à Callisulfan 350 EC (endosulfan $700 \mathrm{~g} / \mathrm{ha}$ ).

A la récolte du coton graine, sur la station de Savalou, le profenofos quelle que soit son origine, procure un rendement significativement supérieur $(\mathrm{p}<0,05)$ à l'endosulfan (Tableau 5). En milieu paysan, le Calfos $500 \mathrm{EC}$ (profenofos $750 \mathrm{~g} / \mathrm{ha}$ ) a fournit un rendement équivalent à celui de Callisulfan 350 EC (endosulfan $700 \mathrm{~g} / \mathrm{ha}$ ). La performance de Fanga 500 EC en milieu réel n'a pas été celle qu'on attendait, car, quand bien même aucune différence significative n'a été observée entre le Fanga 500 EC et le Calfos 500 EC, le rendement obtenu avec l'application de Fanga 500 EC est significativement inférieur $(p<0,05)$ au rendement fourni par l'utilisation de Callisulfan 350 EC.

Tableau 1 : $\quad$ Nombres de différentes chenilles carpophages par are durant l'essai sur la station d'expérimentation de Sékou.

\begin{tabular}{|c|c|c|c|c|c|c|c|c|}
\hline \multirow[t]{3}{*}{ Produits } & \multicolumn{8}{|c|}{ Nombre de chenilles par are } \\
\hline & \multicolumn{3}{|c|}{ Helicoverpa armigera } & \multicolumn{3}{|c|}{ Pectinophora gossypiella } & \multicolumn{2}{|c|}{ Earias spp } \\
\hline & $\begin{array}{l}3^{\text {ème }} \\
\text { Obs }\end{array}$ & $\begin{array}{l}7^{\mathrm{ème}} \\
\text { Obs }\end{array}$ & $\begin{array}{c}\text { Cumul } \\
\text { (12 obs) }\end{array}$ & $\begin{array}{l}3^{\text {ème }} \\
\text { Obs }\end{array}$ & $\begin{array}{l}8^{\text {ème }} \\
\text { obs }\end{array}$ & $\begin{array}{c}\text { Cumul } \\
(12 \text { obs })\end{array}$ & $\begin{array}{l}2^{\mathrm{ème}} \\
\text { obs }\end{array}$ & $\begin{array}{c}\text { Cumul } \\
(12 \text { obs }) \\
\end{array}$ \\
\hline $350 \mathrm{EC}$ & $0 \mathrm{a}$ & $42 \mathrm{~b}$ & $115 \mathrm{bc}$ & $17 \mathrm{bc}$ & $0 \mathrm{a}$ & $139 \mathrm{~b}$ & $38 \mathrm{~b}$ & $198 \mathrm{~b}$ \\
\hline $330 \mathrm{CS}$ & $0 \mathrm{a}$ & $10 \mathrm{a}$ & $45 \mathrm{a}$ & $0 \mathrm{a}$ & $10 \mathrm{a}$ & $56 \mathrm{a}$ & $28 \mathrm{ab}$ & $80 \mathrm{a}$ \\
\hline Calfos $500 \mathrm{EC}$ & $0 \mathrm{a}$ & $3 \mathrm{a}$ & $66 \mathrm{ab}$ & $7 \mathrm{ab}$ & $7 \mathrm{a}$ & $66 \mathrm{a}$ & $10 \mathrm{a}$ & $111 \mathrm{a}$ \\
\hline Thiofanex 350 EC & $24 \mathrm{~b}$ & $10 \mathrm{a}$ & $132 \mathrm{c}$ & $35 \mathrm{c}$ & $31 \mathrm{~b}$ & $108 \mathrm{ab}$ & $42 \mathrm{~b}$ & $108 \mathrm{a}$ \\
\hline
\end{tabular}

Obs = observation ; Les observations réalisées à des dates ponctuelles ne sont pas mentionnées si elles ne sont pas discriminantes. Par exemple la quatrième observation pour le dénombrement de Helicoverpa armigera n'est pas présentée. Les valeurs possédant la même lettre a, b ou c ne sont pas significativement différentes par colonne. 
Tableau 2: Nombre de capsules vertes saines et trouées durant l'essai sur la station d'expérimentation de Sékou.

\begin{tabular}{|c|c|c|c|c|c|c|c|}
\hline \multirow[b]{3}{*}{ Produits } & \multicolumn{7}{|c|}{ Pourcentage de capsules vertes } \\
\hline & \multicolumn{3}{|c|}{ Saines } & \multicolumn{4}{|c|}{ Trouées } \\
\hline & $\begin{array}{l}1^{\text {ère }} \\
\text { Obs }\end{array}$ & $\begin{array}{l}7^{\text {ème }} \\
\text { Obs }\end{array}$ & $\begin{array}{c}\text { Moyenne } \\
(8 \text { obs })\end{array}$ & $\begin{array}{l}1^{\text {ère }} \\
\text { obs }\end{array}$ & $\begin{array}{l}5^{\text {ème }} \\
\text { obs }\end{array}$ & $\begin{array}{l}7^{\grave{e ̀ m e}} \\
\text { Obs }\end{array}$ & $\begin{array}{c}\text { Moyenne } \\
(8 \text { obs })\end{array}$ \\
\hline $350 \mathrm{EC}$ & $76,3 \mathrm{ab}$ & $73,5 \mathrm{a}$ & $69,2 \mathrm{a}$ & $10,7 \mathrm{a}$ & $25,2 \mathrm{~b}$ & $5,8 \mathrm{a}$ & $15,0 \mathrm{ab}$ \\
\hline $330 \mathrm{CS}$ & $79,0 \mathrm{a}$ & 69,3 a & $68,8 \mathrm{a}$ & $10,5 \mathrm{a}$ & $21,2 \mathrm{ab}$ & $6,3 \mathrm{a}$ & 13,6 a \\
\hline Calfos $500 \mathrm{EC}$ & $73,2 \mathrm{ab}$ & $75,3 \mathrm{a}$ & $66,0 \mathrm{ab}$ & $14,7 \mathrm{ab}$ & $25,7 \mathrm{~b}$ & $4,3 \mathrm{a}$ & $16,9 \mathrm{~b}$ \\
\hline Thiofanex $350 \mathrm{EC}$ & $72,2 \mathrm{~b}$ & $55,2 \mathrm{~b}$ & $63,4 \mathrm{~b}$ & $17,0 \mathrm{~b}$ & $16,2 \mathrm{a}$ & $14,3 \mathrm{~b}$ & $17,3 \mathrm{~b}$ \\
\hline
\end{tabular}

Obs = observation ; Les observations réalisées à des dates ponctuelles ne sont pas mentionnées si elles ne sont pas discriminantes. Par exemple la deuxième observation des capsules vertes saines n'est pas présentée. Les valeurs possédant la même lettre a ou b ne sont pas significativement différentes par colonne.

Tableau 3 : $\quad$ Nombre de capsules vertes piquées, nombre de capsules mûres tachées, nombre de chenilles dans 100 capsules vertes et rendement de coton graine durant l'essai sur la station d'expérimentation de Sékou.

\begin{tabular}{|c|c|c|c|c|c|c|c|c|c|}
\hline \multirow{3}{*}{ Produits } & \multirow{2}{*}{\multicolumn{2}{|c|}{$\begin{array}{c}\text { Pourcentage de } \\
\text { capsules vertes } \\
\begin{array}{c}\text { Piquées par des } \\
\text { punaises }\end{array}\end{array}$}} & \multicolumn{5}{|c|}{ Chenilles dans 100 capsules vertes } & \multirow{2}{*}{$\begin{array}{c}\% \\
\text { capsules } \\
\text { mûres } \\
\text { tachées }\end{array}$} & \multirow{2}{*}{$\begin{array}{c}\text { Rdt en } \\
\text { coton } \\
\text { graine } \\
\text { (kg/ha) }\end{array}$} \\
\hline & & & \multicolumn{2}{|c|}{ P. gossypiella } & \multirow{2}{*}{$\begin{array}{c}\begin{array}{c}\text { Earias } \\
\text { spp }\end{array} \\
\text { Moy } \\
(8 \text { obs })\end{array}$} & \multicolumn{2}{|c|}{ C. leucotreta } & & \\
\hline & $\begin{array}{l}5^{\text {ème }} \\
\text { obs }\end{array}$ & $\begin{array}{c}\text { Moy } \\
\text { (8 obs) }\end{array}$ & $\begin{array}{l}7^{\text {ème }} \\
\text { obs }\end{array}$ & $\begin{array}{c}\text { Moy } \\
(8 \text { obs })\end{array}$ & & $\begin{array}{l}6^{\text {ème }} \\
\text { obs }\end{array}$ & $\begin{array}{l}\text { Moy } \\
\text { (8obs) }\end{array}$ & $\begin{array}{c}\text { Moy } \\
\text { (3 obs) }\end{array}$ & $\begin{array}{c}\text { Total } \\
(3 \text { obs })\end{array}$ \\
\hline $350 \mathrm{EC}$ & $11,2 \mathrm{a}$ & $15,6 \mathrm{a}$ & $0,8 \mathrm{~b}$ & $0,4 \mathrm{a}$ & $2,5 \mathrm{~b}$ & $9,3 \mathrm{~b}$ & $8,1 \mathrm{a}$ & $54,6 \mathrm{~b}$ & 1113 \\
\hline $330 \mathrm{CS}$ & $15,3 \mathrm{a}$ & $17,5 \mathrm{ab}$ & $0,2 \mathrm{a}$ & $0,2 \mathrm{a}$ & $1,9 \mathrm{ab}$ & $4,7 \mathrm{a}$ & $7,5 \mathrm{a}$ & $36,2 \mathrm{a}$ & 995 \\
\hline Calfos $500 \mathrm{EC}$ & $14,7 \mathrm{a}$ & $16,9 \mathrm{ab}$ & $0,0 \mathrm{a}$ & $0,6 \mathrm{~b}$ & $1,6 \mathrm{ab}$ & $7,5 \mathrm{ab}$ & $8,7 \mathrm{a}$ & $48,1 \mathrm{ab}$ & 1025 \\
\hline Thiofanex $350 \mathrm{EC}$ & $24,7 \mathrm{~b}$ & $19,3 \mathrm{~b}$ & $0,0 \mathrm{a}$ & $0,2 \mathrm{a}$ & $1,5 \mathrm{a}$ & $6,8 \mathrm{ab}$ & $10,4 \mathrm{~b}$ & $47,1 \mathrm{ab}$ & 899 \\
\hline
\end{tabular}

Obs = observation $; \%=$ pourcentage $;$ Moy = moyenne $; \mathrm{Rdt}=$ Rendement $;$ Les observations réalisées à des dates ponctuelles ne sont pas mentionnées si elles ne sont pas discriminantes. Par exemple la sixième observation des capsules vertes piquées par des punaises n'est pas présentée. Les valeurs possédant la même lettre a ou b ne sont pas significativement différentes par colonne.

Tableau 4: Plants et feuilles attaqués par A.gossypii et nombres de chenilles de $P$. gossypiella par are durant l'essai sur la station d'expérimentation de Savalou.

\begin{tabular}{|c|c|c|c|c|c|c|c|c|c|}
\hline \multirow[t]{2}{*}{ Produits } & \multicolumn{2}{|c|}{$\begin{array}{l}\text { Nombre de chenilles } P \text {. } \\
\text { gossypiella par are }\end{array}$} & \multicolumn{3}{|c|}{$\begin{array}{c}\text { plants attaqués par } A . \\
\text { gossypii (en \%) }\end{array}$} & \multicolumn{4}{|c|}{$\begin{array}{c}\text { Feuilles attaquées par } A . \text { gossypii (en } \\
\%)\end{array}$} \\
\hline & $5^{\text {ème }}$ obs & $\begin{array}{l}\text { Cumul } \\
\text { (11 obs) }\end{array}$ & $5^{\text {ème }}$ obs & $6^{\text {ème }}$ obs & $\begin{array}{c}\text { Moy. } \\
\text { (11 obs) }\end{array}$ & $2^{\text {ème }}$ obs & $3^{\text {ème }}$ obs & $5^{\text {ème }}$ obs & $\begin{array}{c}\text { Moy } \\
\text { (11 obs) }\end{array}$ \\
\hline Callisulfan 350 & $63 \mathrm{~b}$ & $354 \mathrm{~b}$ & $11 \mathrm{~b}$ & $4 \mathrm{~b}$ & $7 \mathrm{c}$ & $17,0 \mathrm{~b}$ & $36,5 \mathrm{~b}$ & $8,5 \mathrm{~b}$ & $9,6 \mathrm{c}$ \\
\hline Calfos 500 EC & $0 \mathrm{a}$ & $52 \mathrm{a}$ & $1 \mathrm{a}$ & $0 \mathrm{a}$ & $5 \mathrm{~b}$ & $3,8 \mathrm{a}$ & $18,2 \mathrm{ab}$ & $0,0 \mathrm{a}$ & $5,1 \mathrm{~b}$ \\
\hline Fanga 500 EC & $7 \mathrm{a}$ & $38 \mathrm{a}$ & $1 \mathrm{a}$ & $0 \mathrm{a}$ & $3 a$ & $0,2 \mathrm{a}$ & $0,0 \mathrm{a}$ & $0,0 \mathrm{a}$ & $2,2 \mathrm{a}$ \\
\hline
\end{tabular}

Obs = observation ; Moy = Moyenne , Les observations réalisées à des dates ponctuelles ne sont pas mentionnées si elles ne sont pas discriminantes. Par exemple la septième observation de plants attaqués par A. gossypii n'est pas présentée. Les valeurs possédant la même lettre $\mathrm{a}, \mathrm{b}$ ou $\mathrm{c}$ ne sont pas significativement différentes par colonne. 
Tableau 5: Nombre de chenilles dans 100 capsules vertes durant l'essai sur la station d'expérimentation de Savalou et rendement de coton-graine en station et en milieu réel.

\begin{tabular}{|c|c|c|c|c|c|c|c|c|}
\hline \multirow{3}{*}{ Produits } & \multicolumn{6}{|c|}{$\begin{array}{c}\text { Nombre de chenilles dans } 100 \text { capsules } \\
\end{array}$} & \multirow{3}{*}{$\begin{array}{c}\begin{array}{c}\text { Rendement sur } \\
\text { station } \\
(\mathbf{k g} / \mathrm{ha})\end{array} \\
\begin{array}{c}\text { Total } \\
\text { (3 récoltes) }\end{array}\end{array}$} & \multirow{3}{*}{$\begin{array}{c}\text { Rendement } \\
\text { en milieu } \\
\text { Réel } \\
(\mathbf{k g} / \mathbf{h a})\end{array}$} \\
\hline & \multicolumn{3}{|c|}{ H. armigera Earias spp C. leucotreta } & \multicolumn{3}{|c|}{ P. gossypiella } & & \\
\hline & $\begin{array}{c}\text { Moy } \\
\text { (8 obs) }\end{array}$ & $\begin{array}{l}\text { Moy } \\
\text { (8 obs) }\end{array}$ & $\begin{array}{l}\text { Moy } \\
\text { (8 obs) }\end{array}$ & $\begin{array}{l}6^{\text {ème }} \\
\text { obs }\end{array}$ & $\begin{array}{l}8^{\text {ème }} \\
\text { obs }\end{array}$ & $\begin{array}{c}\text { Moy } \\
\text { (8 obs) }\end{array}$ & & \\
\hline Callisulfan 350 & 0,17 & $0,58 \mathrm{~b}$ & 8,17 & $4,50 \mathrm{~b}$ & $4,00 \mathrm{~b}$ & 3,46 & $802 \mathrm{~b}$ & $1814 \mathrm{a}$ \\
\hline Calfos $500 \mathrm{EC}$ & 0,04 & $0,27 \mathrm{ab}$ & 9,83 & $3,33 \mathrm{ab}$ & $4,50 \mathrm{~b}$ & 3,40 & $1079 \mathrm{a}$ & $1672 \mathrm{ab}$ \\
\hline Fanga $500 \mathrm{EC}$ & 0,04 & $0,06 \mathrm{a}$ & 9,19 & $1,00 \mathrm{a}$ & $0,50 \mathrm{a}$ & 3,13 & $1197 \mathrm{a}$ & $1583 \mathrm{~b}$ \\
\hline
\end{tabular}

\section{DISCUSSION}

Sur la station d'expérimentation, dans le Sud Bénin à Sékou et dans le Centre à Savalou, l'application du profénofos comparé à l'endosulfan assure une bonne protection phytosanitaire du cotonnier avec la maîtrise des populations des ravageurs rencontrés notamment $H$. armigera, $P$. gossypiella, Earias spp, C. leucotreta, A. gossypii. Des résultats similaires ont été obtenus par Kathuria et al. (2005) qui ont montré une bonne efficacité du profenofos comparé à l'endosulfan appliqué sur les œufs de $H$. armigera. En Iran, une bonne efficacité du profenofos contre $H$. armigera a été également signalée par Dastjerdi et al. (2008) lors de la réalisation des bioessais au laboratoire. En Côte d'Ivoire, aucune différence significative n'a été observée entre l'endosulfan et le profenofos pour la maîtrise des populations de $H$. armigera en culture cotonnière (Ochou et Martin, 2002).

L'efficacité du profenofos en comparaison avec l'endosulfan a permis d'obtenir sur la station de Savalou une amélioration significative du rendement de coton graine produit. En milieu réel dans le Centre Bénin à Savalou, aucune différence significative n'a été observée entre l'endosulfan et le profenofos (Calfos 500 EC). Le profenofos apparaît au Bénin comme un alternatif à l'endosulfan au Sud et au Centre du pays. Les résultats obtenus dans le Sud et le Centre Bénin durant ces essais ne contredisent pas ceux obtenus par Katary et Djihinto (2007) qui ont mis en relief dans le Nord du Bénin le bon comportement du profenofos comparé à l'endosulfan avec un rendement de $1993 \mathrm{~kg} / \mathrm{ha}$ de coton graine pour l'application du profenofos contre $1899 \mathrm{~kg} / \mathrm{ha}$ de coton graine pour l'utilisation d'endosulfan. Un autre essai de comparaison du profenofos et de l'endosulfan montrait qu'aucune différence significative n'a été observée entre les deux produits à la récolte du coton graine avec un rendement de 2148 $\mathrm{kg} / \mathrm{ha}$ pour l'application d'endosulfan contre $2180 \mathrm{~kg} / \mathrm{ha}$ pour le profenofos (Djihinto, 2004). Au Bénin, comme en Afrique de l'Ouest et dans plusieurs autres pays dans le monde, le profenofos, à l'instar des molécules comme l'indoxacarb, le spinosad, l'emamectine, le lufenuron et le spirotétramate, peut être utilisé comme une alternative à l'endosulfan (Ochou and Martin, 2002 ; Martin et al., 2002 ; Djihinto, 2004 ; Cheema et al., 2004 ; Kathuria et al., 2005 ; Razaq et al., 2005 ; Katary et Djihinto, 2007 ; Dastjerdi et al., 2008 ; Agbohessi et al., 2012). Il est important de mentionner qu'en période de forte infestation de $H$. armigera en culture cotonnière au Bénin, une baisse d'efficacité du profenofos en comparaison avec l'endosulfan peut être observée avec une perte de production pouvant varier entre 41650 tonnes et 96600 tonnes de coton graine pour une surface cultivée de 350000 hectares (Katary et Djihinto, 2007). Les résultats de nos essais étant obtenus dans les conditions de faible à moyenne infestation de $H$. armigera, en accord avec nos études antérieures (Katary et Djihinto, 2007), le profenofos ne peut donc être considéré comme un alternatif à l'endosulfan au Bénin que dans les conditions d'infestation faible à moyenne du ravageur. 


\section{Conclusion}

Les résultats de la comparaison du profenofos à l'endosulfan dans le Sud et le Centre du Bénin ont montré une bonne efficacité du profenofos contre les principaux ravageurs du cotonnier. Le profenofos est parfois meilleur que l'endosulfan notamment pour le contrôle de $H$. armigera, $P$. gossypiella, Earias spp, C. leucotreta, A. gossypii. Ainsi, au terme de la production, l'efficacité du profenofos comparé à l'endosulfan peut se traduire par un accroissement du rendement de coton graine. Les résultats satisfaisants du profenofos comparé à l'endosulfan au Bénin ont permis de mettre ainsi en évidence le profenofos comme un produit alternatif à l'endosulfan en culture cotonnière. L'élargissement de la gamme des produits alternatifs à l'endosulfan peut être poursuivi au Bénin par l'étude d'efficacité de nouvelles molécules insecticides mises au point par les firmes phytosanitaires.

\section{REMERCIEMENTS}

Nous remercions le Directeur Mensah Guy Apolinaire pour la lecture du manuscrit et les commentaires effectués. Nous remercions également la Coopération Française pour son assistance technique.

\section{CONFLIT D'INTERETS} conflit d'intérêts.

Les auteurs déclarent qu'il n'y a pas de

\section{CONTRIBUTIONS DES AUTEURS}

Les contributions individuelles des auteurs dans le cadre de ce travail sont indiquées comme suit : $\mathrm{CAD}$ a été le principal investigateur et a ecrit le manuscrit; $\mathrm{AF}$ a contribué à l'exécution de l'expérimentation sur station à Sékou, à la collecte des données sur station à Sékou à la préparation du manuscrit. ED a contribué à l'exécution de l'expérimentation sur station à Savalou, à la collecte des données sur station à Savalou, et à la préparation du manuscrit. L'auteur GB a participé à l'exécution de l'expérimentation en milieu réel à Savalou et à la collecte des données en milieu réel à Savalou.

\section{RÉFÉRENCES}

Agbohessi P, Imorou Toko I, Attakpa EY, Kestemont P. 2012. Synthèse des caractéristiques physico-chimiques et effet écotoxicologiques du Tihan 175 OTEQ utilisé dans la protection phytosanitaire du cotonnier au Bénin. International Journal of Biological and Chemical Sciences, 6: 2280-2292. Doi: http://dx.doi.org/10.4314/ijbcs.v6i5.34

Cheema GM, Nasreen A, Ashfaq M. 2004. Application of selective insecticides to control first generation of Helicoverpa armigera (Hubner) on cotton. Pak. Entomol., 26: 35-37.

Dastjerdi HR, Hejazi MJ, Ganbalani GN, Saber M. 2008. Toxicity of some biorational and conventional insecticides to cotton bollworm, Helicoverpa armigera (Lepidoptera : Noctuidae) and its ectoparasitoid, Habrobracon hebetor (Hymenoptera : Braconidae). Journal of Entomological Society of Iran, 28: 2737.

Djihinto CA. 2004. La résistance de Helicoverpa armigera (Hubner, 1808) aux pyréthrinoïdes en culture cotonnière au Bénin: du mécanisme, du coût biologique et des stratégies de gestion de la résistance. Thèse de doctorat unique, Université de Cocody Abidjan, Cocody Abidjan, p. 259.

Djihinto CA, Hougni A, Houngbo EN, Katary A, Bokonon-Ganta HA. 2013. Insecticide resistance fitness cost and resistance stability. International Journal of Tropical Agriculture and Food Systems, 7: 17-29.

Djihinto CA, Katary A, Djaboutou CM, Prudent P, Menozzi P, Atachi P. 2012. Variation in biological parameters of cypermethrin resistant and susceptible strains of Helicoverpa armigera from 
Benin Republic, West Africa. Int. J. Biol. Chem. Sci., 6: 931-940. Doi: http://dx.doi.org/10.4314/ijbcs.v6i3.2

Djihinto CA, Katary A, Prudent P, Vassal JM, Vaissayre M. 2009. Variation in resistance to pyrethroids in Helicoverpa armigera from Benin Republic, West Africa. J. Econ. Entomol., 102: 19281934.

Karim S. 2000. Management of Helicoverpa armigera: A review and prospectus for Pakistan. Pakistan Journal of Biological Sciences, 3: 1213-1222.

Katary A. 2003. Etude spatio-temporelle de la gestion de la résistance de $H$. armigera aux pyréthrinoïdes en culture cotonnière au Bénin. Thèse de Doctorat d'Etat, Université de Cocody Abidjan, Cocody Abidjan, p. 250.

Katary A, Djihinto CA. 2007. Programme fenêtre et gestion de Helicoverpa armigera aux pyréthrinoïdes en cultures cotonnières au Bénin. Bulletin de la Recherche Agronomique du Bénin, 56: 24-35.

Kathuria V, Saini RK, Ram P. 2005. Response of Helicoverpa armigera (Hübner) to different insecticides: a brief account. Resistant Pest Management Newsletter, 14: 2-6.

Martin T, Ochou GO, Vaissayre M, Fournier D. 2002. Positive and negative crossresistance to pyrethroids in Helicoverpa armigera from West Africa. Resistant Pest Management Newsletter, 12: 16-20.

Nimbalkar RK, Shinde SS, Tawar DS, Muley SP. 2009. Response of cotton Bollworm Helicoverpa armigera (Hubner) (Lepidoptera: noctuidae) to different insecticides in Maharashtra, India. World Journal of Agricultural Sciences, 5: 250255.

Ochou GO, Martin T. 2002. Pyrethroid resistance in Helicoverpa armigera (Hübner): recent developments and prospects for its management in Côte d'Ivoire, West Africa. Resistant Pest Management Newsletter, 12: 10-16.

Prudent P, Loko S, Deybe D, Vaissayre M. 2007. Factors limiting the adoption of IPM practices by cotton farmers in Bénin: a participatory approach. Expl. Agric., 43: 113-124. Doi: 10.1017/S00144797006004261

Razaq M, Suhail A, Aslam M, Arif MJ, Saleem MA, Khan MHA. 2005. Evaluation of new chemistry and conventional insecticides against Helicoverpa armigera (Hubner) on cotton at Multan (Pakistan). Pak. Entomol., 27: 71-73.

Silvie PJ, Renou A, Vodounnon S, Bonni G, Adegnika MO, Héma O, Prudent $P$, Sorèze J, Ochou OG, Togola M, Badiane D, Ndour A, Akantetou PK, Ayeva B, Brévault T. 2013. Threshold-based interventions for cotton pest control in West Africa: what's up 10 years later? Crop Protection, 43: 157-165.

Yaqoob M, Arora RK. 2005. Development of resistance in Helicoverpa armigera (Hubner) to endosulfan in Jammu and comparative biology of its resistant, parental and susceptible strains. Resistant Pest Management Newsletter, 14: 10-13. 